Computer Optics and Nanophotonics

\title{
AVEFRONT ANALYSIS BASED ON ZERNIKE POLYNOMIALS
}

\author{
M.S. Kirilenko ${ }^{1,2}$, P.A. Khorin ${ }^{1}$, A.P. Porfirev ${ }^{1,2}$ \\ ${ }^{1}$ Samara National Research University, Samara, Russia \\ ${ }^{2}$ Image Processing Systems Institute - Branch of the Federal Scientific Research Centre "Crys- \\ tallography and Photonics" of Russian Academy of Sciences, Samara, Russia
}

\begin{abstract}
The simulation of optical wavefront decomposition on Zernike polynomials based on the multi-channel diffractive optical element (DOE) was performed. This approach is an alternative to classical wavefront sensors and may be used to determine weighted values of aberrations in the analysed wavefront in real time thus, facilitating the aberrometry analysis of the human eye optical system. An investigation of multi-channel analysers was performed on test examples and the applicability of the techniques in solving particular applications are defined.
\end{abstract}

Keywords: wavefront analysis, Zernike polynomials, human eye optical system, multi-channel diffractive optical element.

Citation: Kirilenko MS, Khorin PA, Porfirev AP. Wavefront analysis based on Zernike polynomials. CEUR Workshop Proceedings, 2016; 1638: 66-75. DOI: 10.18287/1613-0073-2016-1638-66-75

\section{Introduction}

Wavefront analysis at the output of the human eye optical system is difficult [1-3]. Wavefront analysis based on an expansion in the Zernike basis facilitates diagnosis in clinical ophthalmology. It allows the creation of the most advanced lenses, presently providing the highest quality vision. Multi-order diffractive optical elements (DOE) are used for the direct measurement of the amplitude of the optical wavefront expansion coefficients on Zernike polynomials.

Multi-order (multi-channel) DOE, based on the superposition of orthogonal functions, allows the coefficients of the analyzed field expansion in the used basis to be spatially divided [4-6]. Multi-order DOE with Zernike polynomials can be used for wavefront analysis and wavefront reconstruction [7, 8], including aberrometry analysis of the human eye optical system [9].

The human eye can be described as a system of lenses consisting of three basic components: the cornea, pupil and lens. The cornea (including the tear film) structure is the dominant eye optical power (it provides about $70 \%$ of the optical power of the eye). Accordingly, it is a major source of aberrations in the eye. The front surface of 
the cornea has an elongated profile; that is, the central region is steeper than at the periphery. This shape helps reduce the amount of spherical aberration in the whole eye. However, the cornea shape may be significantly different for different people, and this results in asymmetric astigmatism and high-order aberration (for example, coma). Optical elements of the human eye optical systems work concertedly to create an image on the retina. However, the image in the real system of a human eye is never perfect. The emergence of additional optical aberrations associated with aging or disease degrades the image quality significantly. To compensate the wavefront distortions, it is necessary to determine exactly which aberration led to these distortions.

\section{Wavefront analysis techniques. Basis of Zernike polynomials}

Currently, the most widely used technique for the aberrometry analysis of the human eye optical system is the Shack-Hartmann wavefront sensor[10,11]. The sensor consists of an array of microlenses optically conjugated to the pupil of the eye, with the camera located at the focal plane of the lenses. Flat wavefront looks at the camera as an absolute correct grid of light spots. However, if the analysed wavefront is distorted by aberrations, the light spots are located irregularly. Displacement of each spot from the reference position is proportional to the derivative of the wavefront in each of the microlenses. Thus, the wavefront is reconstructed from the values of the displacement of light spots. Then, aberrations are calculated.

A common representation of the wavefront is a series of Zernike polynomials. Lower polynomials describe defocus and astigmatism. The higher-order coefficients correspond to spherical aberration of oblique rays, which occurs as a result of misalignment of the lens and foveola, as well as deviations in the periphery of the lens [2].

The expansion coefficients of the wavefront in orthogonal Zernike polynomials allow the determination of the mean square error of deviation from the ideal wavefront. The coefficients with high absolute values will automatically point to the aberrations that cause the greatest distortion of the wavefront. Thus, it significantly accelerates and simplifies the analysis of the patient's vision.

In [9], the authors proposed the use of multi-channel DOE [7, 8, 12] matched with a set of Zernike functions for the direct measurement of coefficients of wavefront aberrations of the human eye optical system. Examples of Zernike functions are shown in the Table 1.

Circular Zernike polynomials represent a complete set of orthogonaltional functions with angular harmonics in a circle of radius $R$ :

$\Psi_{n m}(r, \varphi)=\sqrt{\frac{n+1}{\pi R^{2}}} Z_{n}^{m}(r) \exp (\operatorname{im} \varphi)$,

where $Z_{n}^{m}(r)$ are the radial Zernike polynomials: 
$Z_{n}^{m}(r)=\sum_{p=0}^{(n-m) / 2} \frac{(-1)^{p}(n-p) !}{p !\left(\frac{n+m}{2}-p\right) !\left(\frac{n-m}{2}-p\right) !}\left(\frac{r}{R}\right)^{n-2 p}$,

$|m| \leq n, \quad(n-m)$ is even, $(r, \varphi)$ are the polar coordinates.

Table 1. Examples of Zernike functions

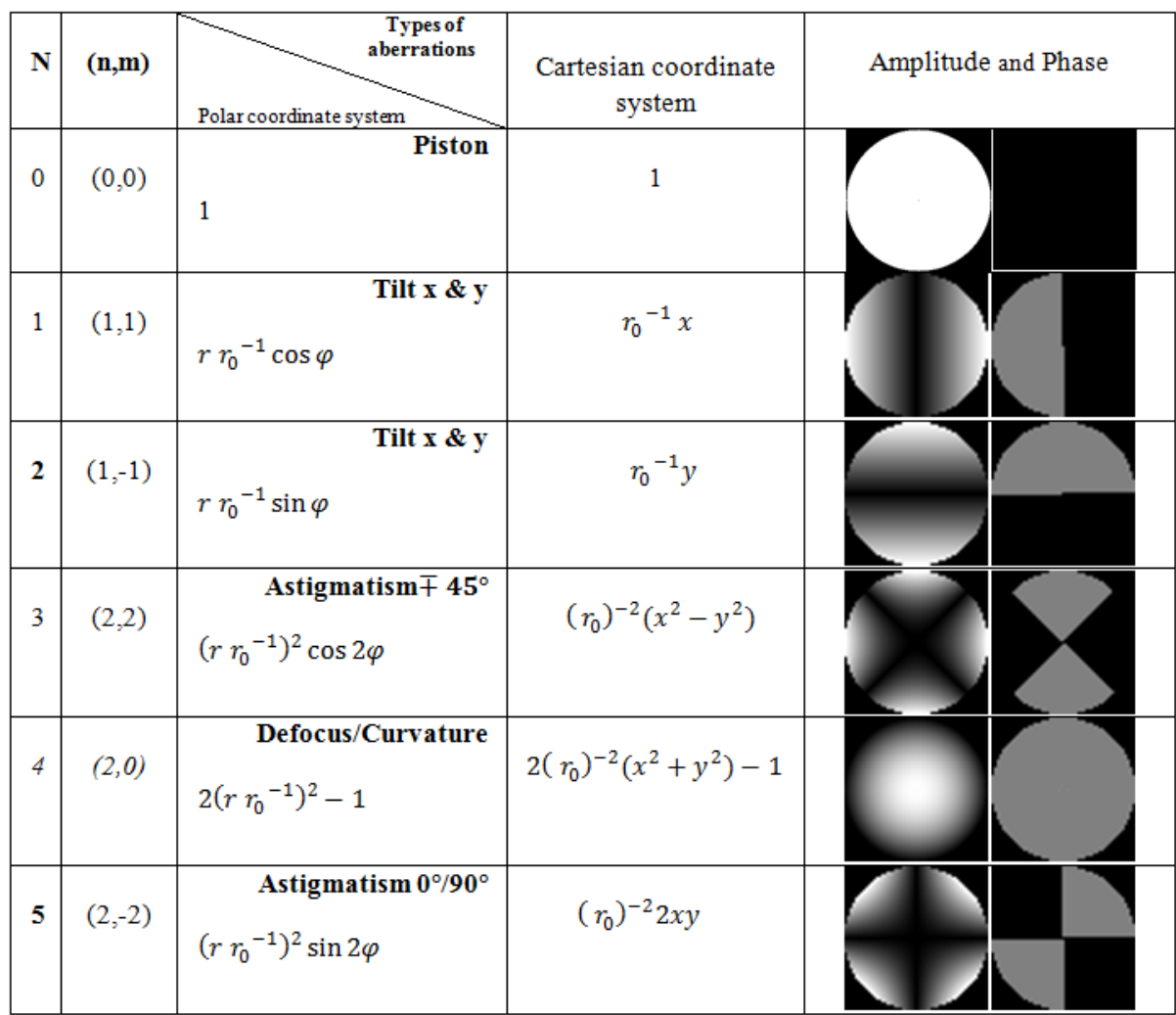

Set of 8 - 10 Zernike functions are usually sufficient for the smooth optics.

\section{The aberrations of the optical structures of the eye}

Lower-order aberrations are the predominant optical aberrations in the optical system of the human eye [13]. They constitute about $90 \%$ of the wave aberrations. Positive (hyperopia) or negative (myopia) defocus is the dominant aberration followed by astigmatism (orthogonal or oblique). In most cases,lower-order aberration can be corrected with glasses, contact lenses or corneal laser surgery.

The development of wavefront sensing techniques allowed accurate and objective measurement of wave aberrations of the eye to be obtained in the clinical setting. 
Coma-like (vertical coma, horizontal coma, oblique trefoil, horizontaltrefoil) and spherical aberration, which are the aberrations of the 3rd order, prevail among the higher-order aberrations in the eye optical system.

In general, the values of the individual lower-order aberrations in the normal eye are scattered randomly around zero with two clear exceptions. The first exception is a spherical aberration, which is systematically displaced towards positive values. The second exception is oblique trefoil which typically has a negative value. The range of absolute values of higher Zernike modes reduces systematically. This means that the amount of aberration coefficients for any person is generally less for the higher-order modes than for the lower-order modes. However, the higher orders can still have a significant negative impact on the quality of the image when the pupil is large.

It is well known that the contribution of higher-order aberrations in the total wave aberration increases with pupil size (average increase is from $3 \%$ to $14 \%$ with increasing pupil size of $3.0 \mathrm{~mm}$ to $7.0 \mathrm{~mm}$ [14]). On the other hand, in eyes with good visual acuity, more visual advantage can be obtained by correcting the higher-order aberrations over the medium and large pupils. This is particularly relevant to spherical aberration. In fact, the relative contribution of the spherical aberration into common wave aberration of the eye increases when the pupil expands and becomes dominant in the higher-order aberrations.

\section{$4 \quad$ Numerical simulation of expansion in Zernike polynomials}

The expansion of the light field with complex amplitude $E(r, \phi)$ into a series in terms of the functions in Eq. (1) is given by:

$$
E(r, \varphi)=\sum_{n=0}^{\infty} \sum_{m=-n}^{n} C_{n m} \Psi_{n m}(r, \varphi)
$$

To find the coefficients $C_{k l}$, we need to multiply Eq. (3) by $\psi_{k l}(r, \varphi)$. Taking into account the orthogonality of the functions $\left\{\psi_{k l}(r, \varphi)\right\}$, all terms of the series turn to zero, except for the term when $n=\mathrm{k}, m=1$ :

$$
\begin{aligned}
& C_{k l}=\frac{\left\langle E(r, \varphi), \psi_{k l}(r, \varphi)\right\rangle}{\left\|\psi_{k l}(r, \varphi)\right\|^{2}}, \\
& \left\langle E(r, \varphi), \psi_{k l}(r, \varphi)\right\rangle=\int_{0}^{2 \pi} \int_{0}^{r_{0}} E(r, \varphi) \overline{\psi_{k l}(r, \varphi)} r d r d \varphi, \\
& \left\|\psi_{k l}(r, \varphi)\right\|^{2}=\left\langle\psi_{k l}(r, \varphi), \psi_{k l}(r, \varphi)\right\rangle=\int_{0}^{2 \pi} \int_{0}^{r_{0}} \psi_{k l}(r, \varphi) \overline{\psi_{k l}(r, \varphi)} r d r d \varphi .
\end{aligned}
$$


If the wavefront is represented by a linear combination of Zernike polynomials, it has a number of useful properties. Firstly, Zernike polynomials are easy to relate to classical aberrations. Secondly, polynomials are usually defined by procession of values at the points using ordinary least squares. Consequently, since Zernike polynomials are orthogonal on the unit circle, any of the terms of the expansion are also the best approximations of the ordinary least squares. Thus, to prevent the shift of focus or tilt of the wavefront it is necessary that the corresponding coefficients are equal to zero. The average value of the aberration is determined by the value of each respective member, so it is not necessary to perform a new approximation using ordinary least squares.

Decomposition of some specified fields on the basis of Zernike (see Eq.(1)) functions are performed by Eq.(4).

Fig. 1 shows the amplitude and phase of the field

$E(r, \varphi)=r \exp \left(-\frac{r^{2}}{\sigma^{2}}\right) \exp (i 3 \varphi)$.

(a)
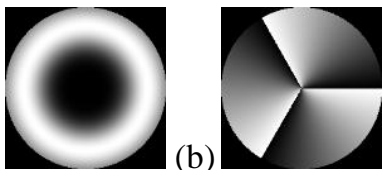

Fig. 1. Amplitude (a) and phase (b) of field described by Eq. (7)

Fig. 2 is a graph showing the distribution of the expansion coefficients for the field described by Eq. (7). It can be seen which aberrations are present in the analysed field and which are equal to their weight. The function describing oblique trefoil $\Psi_{3,3}$ has the largest contribution.

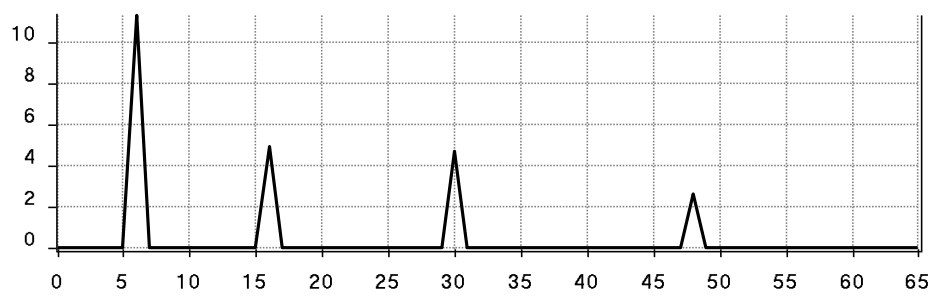

Fig. 2. The distribution of the expansion coefficients for the field described by Eq. (7)

As can be seen, the coefficients of expansion of only those of functions which $m=3$, are non-zero. This is due to the consistency of Zernike basis with optical vortices or angular harmonics [15-19]. 


\section{Simulation of optical expansion of the analysed wavefront}

In order to implement a multi-channel filter, a few basic functions must be coded in different diffraction orders generated by the DOE (see Fig. 3).

$$
\tau(x, y)=\sum_{p=0}^{P} \sum_{q=0}^{Q} \Psi_{p q}^{*}(x, y) \exp \left[i\left(\alpha_{p q} x+\beta_{p q} y\right)\right] .
$$

(a)
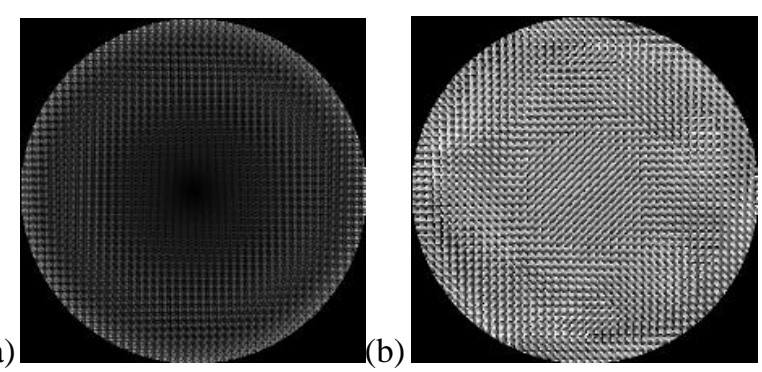

Fig. 3. Amplitude (a) and phase (b) of the 8-th channel DOE

An example of a decomposition planar wavefront $w(x, y)=1$ using the 8 -th channel DOE is shown in Fig. 4. It can be seen, there is no aberration in the analyzed field (null values at the centre of the diffraction orders).

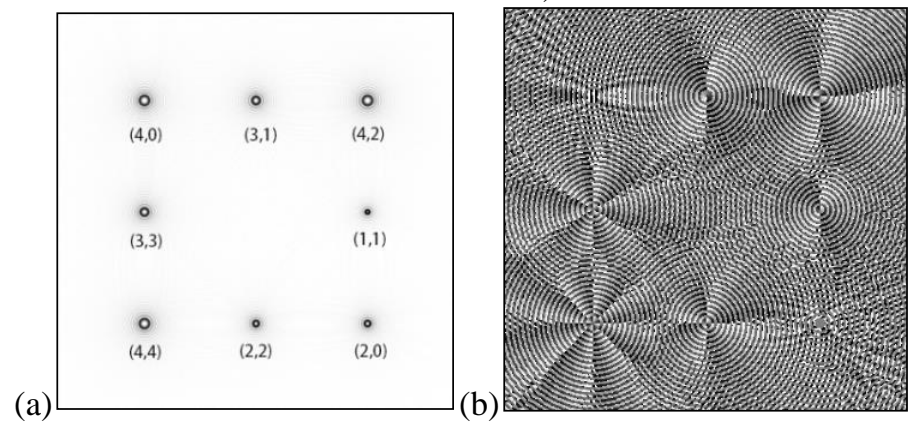

Fig. 4. Generate amplitude (negative) and phase in the focal plane of a lens when illuminated by the 8 -th channel DOE with a plane wave

Figure 6 shows an action of the 8-th channel DOE on a field containing two Zernike functions $w(x, y)=\Psi_{2,0}(x, y)+\Psi_{2,2}(x, y)$ (amplitude and phase are shown in Fig. 5). The aberration of the form $\Psi_{2,0}(x, y), \Psi_{2,2}(x, y)$ was observed $\left(C_{2,0}=C_{2,2}=1\right)$.

The number of channels for the DOE can be significantly increased [17, 18]. Fig. 7 shows the action of a 16-th channel filter. In this case, it is not necessary to use the central region as the information channel.

Similarly, any type of field can be applied to the filter input, and thus it is possible to identify certain aberrations and their weight. 


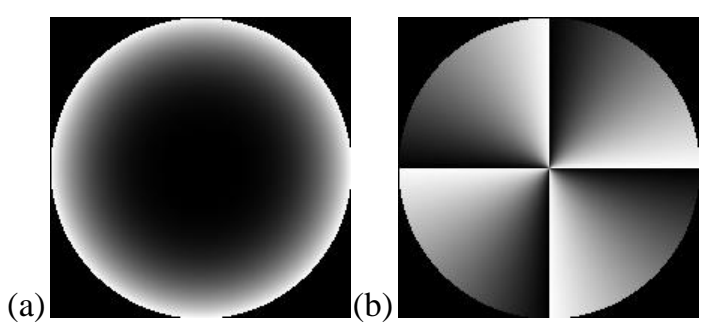

Fig. 5. Amplitude and phase of the analysed field in the form $w(x, y)=\Psi_{2,0}(x, y)+\Psi_{2,2}(x, y)$

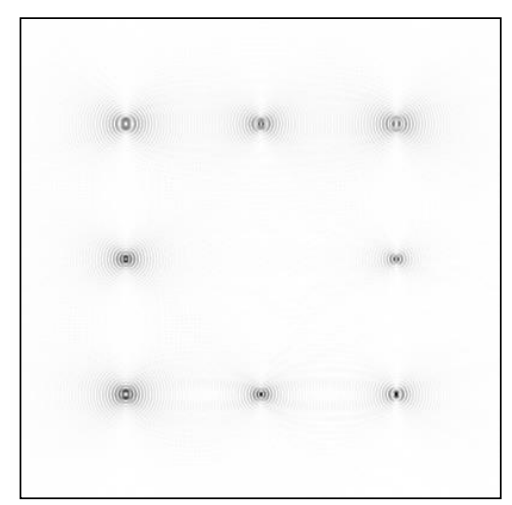

Fig. 6. Action of the 8-th channel DOE for the analyzed field $w(x, y)=\Psi_{2,0}(x, y)+\Psi_{2,2}(x, y)$

\begin{tabular}{|ccccc|}
\hline$\circ$ & & $\circ$ & & $\circ$ \\
$(7,7)$ & & $(6,4)$ & & $(4,2)$ \\
& $\circ$ & $\circ$ & $\circ$ & \\
& $(4,0)$ & $(3,1)$ & $(4,2)$ & \\
$\circ$ & $\circ$ & & $\circ$ & $\circ$ \\
$(6,6)$ & $(3,3)$ & & $(1,1)$ & $(5,5)$ \\
& $\circ$ & $\circ$ & $\circ$ & \\
& $(4,4)$ & $(2,2)$ & $(2,0)$ & \\
$\circ$ & & $\circ$ & & $\circ$ \\
$(6,2)$ & & $(5,3)$ & & $(6,0)$ \\
\hline
\end{tabular}

Fig. 7. Action of the 8-th channel DOE when illuminated the 8-th channel DOE with a plane wave

\section{Experimental investigation}

Figure 8 shows the optical setup used in our experiments. A system composed of a lens $L_{l}\left(f_{l}=350 \mathrm{~mm}\right)$, and a pinhole $(40 \mu \mathrm{m}$ aperture $)$ was utilized for expansion and 
filtration of input laser beam. Then the laser beam incident on the diffractive optical element $D O E_{1}$, generating a Hermite-Gaussian mode $T E M_{10}$. The generated beam illuminated the diffractive optical element $D O E_{2}$. In the first series of experiments, in order to analyze the wavefront generated by $D O E_{1}$, we used 20-th order diffractive optical element generating 10 pairs of Zernike functions as the $D O E_{2}$, in the second series of experiments we used 25-th order diffractive optical element generating 9 pairs of Zernike functions and their combinations. Figure 9 shows the phase transmission functions of diffractive optical elements used in experiments The video-camera Cam LOMO TC-1000 (pixel size is $1.67 \times 1.67 \mathrm{~mm}$ ) was used for shooting the intensity distributions generated in the focal plane of the lens $L_{2}\left(f_{2}=150 \mathrm{~mm}\right)$.

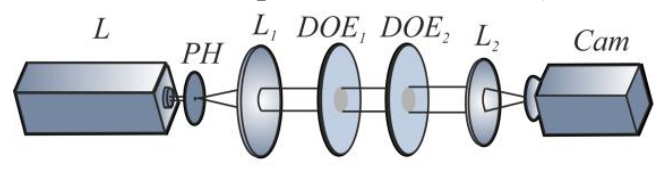

Fig. 8. Experimental optical setup:: $L$ is a linearly polarized laser $(\lambda=633 \mathrm{~nm}), L_{l}$ and $L_{2}$ are lenses $\left(f_{1}=150 \mathrm{~mm}, f_{2}=150 \mathrm{~mm}\right), \mathrm{PH}$ is a pinhole $(40 \mu \mathrm{m}), D O E_{1}$ is a diffractive optical element to generate the Hermite-Gaussian mode $T E M_{10}, D O E_{2}$ is diffractive optical element matched with the basis of Zernike polynomials, Cam is a videocamera LOMO TC-1000 (the pixel size is $1.67 \times 1.67 \mu \mathrm{m}$ )
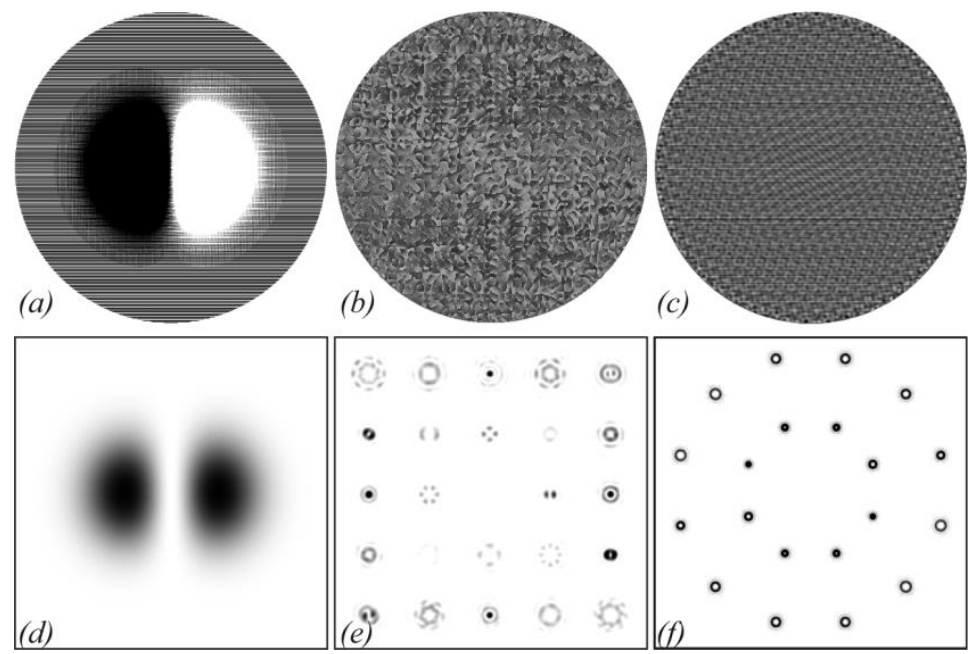

Fig. 9. The phase transmission functions of the diffractive optical elements utilized in experiments (top row), and generated intensity distributions in far-field (negative): (a d) DOE generated Hermite-Gaussian mode $T E M_{10}$, (b, e) 25-th order phase Zernike filter, (c, f) 20-th order phase Zernike filter

Figure 10 shows the intensity distribution experimentally generated by 25 -th order phase Zernike filter illuminated by plane wave and by Hermite-Gaussian mode $T E M_{10}$.Figure 11 shows the intensity distribution experimentally generated by 20 -th order phase Zernike filter illuminated by plane wave and by Hermite-Gaussian mode TEM $_{10}$. 

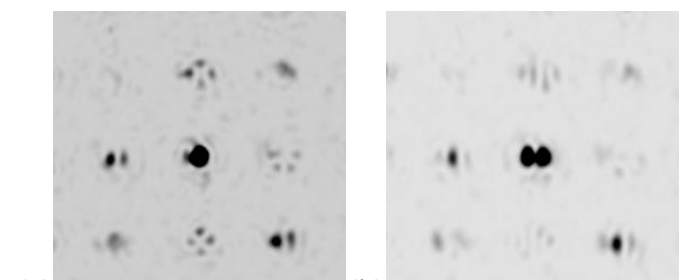

(a)

(b)

Fig. 10. Intensity distributions (negative) experimentally generated by 25 -th order phase Zernike filter illuminated by plane wave (a) and by Hermite-Gaussian mode TEM $_{10}$

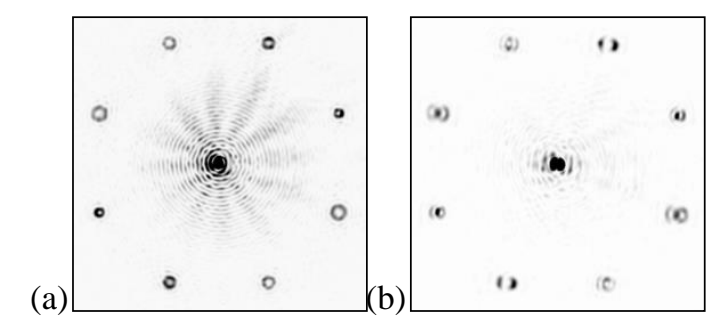

Fig. 11. Intensity distributions (negative) experimentally generated by 20 -th order phase Zernike filter illuminated by plane wave (a) and by Hermite-Gaussian mode TEM $_{10}$

\section{Conclusion}

It is enough to create a field which is the complex conjugate to the identified aberrations to compensate for the most pronounced aberrations identified at the field expansion in the Zernike basis. Such a field can be created by diffractive optic methods [6, 20], including the creation of the corresponding diffraction pattern on the surface of the lens.

Thus, it has been shown that wavefront analysis based on the Zernike polynomials can facilitate a diagnosis in clinical ophthalmology [21].

\section{Acknowledgment}

This work was financially supported by the Russian Foundation for Basic Research (RFBR) (grant 15-29-03823-ofi_m).

\section{References}

1. Smirnov MS. Measurement of the wave aberration of the human eye. Biofizika, 1961; 6: 776- 795 . 
2. Artal P. Optics of the eye and its impact in vision: a tutorial. Advances in Optics and Photonics, 2014; 6: 340-367.

3. Degtyarev SA, Karsakov AV, Branchevskaya ES, Khonina SN, Kotlyar VV. Influence of eye refractive surface curvature modification on the retinal image quality in the LiouBrennan eye model. Computer Optics, 2015; 39(5): 702-708. DOI: 10.18287/0134-24522015-39-5-702-708.

4. Khonina SN, Almazov AA. Design of multi-channel phase spatial filter for selection of Gauss-Laguerre laser modes. Proceedings of SPIE, 2002; 4705: 30-39.

5. Koltyar VV, Khonina SN. Multi-order diffractive optical elements to process data.In Book "Perspectives in Engineering Optics", ed. by K. Singh, V.K. Rastogi. Delhi: Publ. Anita Publications, 2003; 47-56.

6. Golovashkin DL, Kotlyar VV, Soifer VA, Doskolovich LL, Kazanskiy NL, Pavelyev VS, Khonina SN, Skidanov RV. Computer design of diffractive optics. Edited by V.A. Soifer. Cambridge Inter. Scien. Pub. Ltd.\&Woodhead Pub. Ltd., 2012: 896 p.

7. Kotlyar VV, Khonina SN, Soifer VA, Wang Y, Zhao D. Coherent field phase retrieval using a phase Zernike filter. Computer Optics, 1997; 17: 43-48.

8. Ha Y, Zhao D, Wang Y, Kotlyar VV, Khonina SN, Soifer VA. Diffractive optical element for Zernike decomposition. Proceedings of SPIE, 1998; 3557: 191-197.

9. Khonina SN, Kotlyar VV, Kirsh DV. Zernike phase spatial filter for measuring the aberrations of the optical structures of the eye. Journal of Biomedical Photonics Engineering, 2013; 1(2): 146-153.

10. Artzner G. Microlens arrays for Shack-Hartmann wavefront sensors. Optical Engineering, 1992; 31(6): 1311-1322.

11. Liang J, Grimm B, Goelz S, Bille JF. Objective measurement of the WA's aberration of the human eye with the use of a Hartmann-Shack sensor. Journal of the Optical Society of America A, 1994; 11: 1949-1957.

12. Porfirev AP, Khonina SN. Experimental investigation of multi-order diffractive optical elements fitted with the basis of two types of Zernike polynomials. Proceedings of SPIE, 2010, 9807: 98070E.

13. Westheimer G, Liang J. Influence of ocular light scatter on the eye's optical performance. Journal of the Optical Society of America A, 1995; 12: 1417-1424.

14. Lombardo M, Lombardo G, Serrao S. Interocular high-order corneal wavefront aberration symmetry. Journal of the Optical Society of America A, 2006; 23: 777-787.

15. Kotlyar VV, Khonina SN, Soifer VA. Light field decomposition in angular harmonics by means of diffractive optics. Journal of Modern Optics, 1998; 45(7): 1495-1506.

16. Soskin MS, Vasnetov MV. Singular optics. Progress in Optics, 2001; 42: 219-276.

17. Khonina SN, Kotlyar VV, Soifer VA, Paakkonen P, Simonen J, Turunen J. An analysis of the angular momentum of a light field in terms of angular harmonics. Journal of Modern Optics, 2001; 48(10): 1543-1557.

18. Khonina SN, Kotlyar VV, Soifer VA, Jefimovs K, Turunen J. Generation and selection of laser beams represented by a superposition of two angular harmonics. Journal of Modern Optics, 2004; 51(5): 761-773.

19. Kirilenko MS, Khonina SN. Information transmission using optical vortices. Optical Memory and Neural Networks, 2013; 22(2): 81-89.

20. Kotlyar VV, Soifer VA, Khonina SN. Rotation of Gauss-Laguerre multimodal light beams in free space. Technical Physics Letters, 1997; 23 (9): 657-658.

21. Lombardo M, Lombardo G. Wave aberration of human eyes and new descriptors of image optical quality and visual performance. Journal of Cataract and Refractive Surgery, 2010; 36: 313-331. 